\title{
Structural modeling of a forest cluster using discrete mathematics
}

\author{
Olga Sushko, ${ }^{1,}$, Nadezhda Shilovskay ${ }^{1}$, Alexander Plastinin ${ }^{1}$, and Evgeny Shirshov ${ }^{1}$ \\ ${ }^{1}$ Northern (Arctic) Federal University named after M.V. Lomonosov, Severnaya Dvina Emb., 17, \\ Arkhangelsk, 163000, Russia
}

\begin{abstract}
Economic modeling allows studying the development trends of clusters and planning the introduction of control actions in this socioeconomic system to achieve the necessary stabilization and development trends. The cluster can be described as a set of information about its elements and the relationships between them and can be represented as a graph, where the vertices are the agents, and the directed edges are connections between them. Cluster structural formations can be considered as social graphs containing information about heterogeneous factors and the relations between them. Complex structural modeling of the forest cluster allows creating a formal representation of the tasks of identifying the objects (socio-economic systems), analyzing their complexity, coherence, stability, and development scenarios. To do this, we applied the apparatus of discrete mathematics. Signed graphs make it possible to formally make forecasts of the development or trajectory of the simulated system in the phase space of its variables (factors) based on information about its structure and development programs by means of approximating them with pieces of trajectories of impulse processes in signed digraphs. Modeling with the help of the graph theory makes it possible to remove uncertainty associated with predicting the development of a complex system and to propose the option of controlling a stochastic process.
\end{abstract}

\section{Introduction}

\subsection{The relevance of the study of socio-economic systems}

Organizational, economic, social, and political systems are characterized by a large number of elements, relationships between them and the environment, the presence of an uncertainty factor (lack of complete information about their functioning, inaccuracy in quantitative and qualitative estimates of system parameters), different levels, uneven development, and non-linear processes. Therefore, problems of predicting the development of complex systems and their management are classified as poorly structured.

Transformation processes of socio-economic systems (for example, industrial clusters) and the conditions for their development, the ultimate goal of which is their most effective functioning, are associated with significant difficulties. They require, for example, large-

\footnotetext{
${ }^{*}$ Corresponding author: osushko@mail.ru
} 
scale structural changes, reconstruction and modernization of production processes, the transition to new technologies and relationships that satisfy the needs for high-tech and high-quality products that are competitive in the world market, as well as a thorough scientific analysis of the effectiveness of the use of material, financial and public resources. As a result, rapid structural changes in socio-economic relations, the scale of the measures taken, the spectrum of their possible consequences, the introduction of information technologies based on automated systems in all areas of practical activity, necessitate the creation of more effective means of analyzing complex objects and choosing targeted ways for their strategic development.

The most important task of managing socio-economic systems, for example, industrial clusters, is not only the analysis of the present, but also forecasting their future development paths based on the assessment of economic development trends. Socioeconomic forecasting refers to scientifically based predictions of the main trends in socioeconomic, innovative, environmental and territorial development in the long term, the identification of factors that determine the trends in order to justify the strategic and tactical decisions made at the state and corporate levels, allowing uniting social and economic components of the system and considering them in interaction. Economic and mathematical modeling is an effective analysis tool [1].

Economic modeling allows studying the trends of cluster development and planning the introduction of control actions in this socio-economic system to achieve the necessary stabilization and development trends.

\subsection{The approach to modeling a cluster as an economic system}

In the context of modern digitalization, any complex system, for example, a cluster system can be described as a collection of information about its elements and the relationship between them. Such a system consists of many agents (production organizations, financial and scientific institutions) and a set of relations between them and can be represented as a graph, where the vertices of the graph are the agents and the directed edges are the connections between them. Cluster structures are communities within these kinds of social graphs. Thus, economic systems, including cluster ones, can be considered as social graphs containing information about heterogeneous factors and the relations between them.

A social graph [2] is an object that is characterized by:

- great component coherence (it is possible to get from almost any agent to another agent through the connection edges; the vertices of the graph are the agents in this model);

- a small number of connections (on average, for graphs of an economic or social system, especially clusters, the average number of transitions that need to be made between the vertices is not large);

- high density of cooperative ties and the presence of communities within the system (with high probability, if the first vertex of the social graph is connected to the second and the second to the third, then the first vertex will also be connected to the third);

- community background.

This approach to modeling an economic system allows taking advantage of the extensive methodological apparatus developed in the framework of the theory of social graphs $[3,4,5]$.

So, complex structural modeling of the forest cluster allows creating a formal representation of the tasks of identifying objects (socio-economic systems), analyzing their complexity, coherence, stability, and development scenarios using the apparatus of discrete mathematics (for example, signed, weighted signed and functional signed graphs, stability theory, theory of impulse processes). A significant advantage of using the apparatus of discrete mathematics is that it works with both qualitative and quantitative data. Moreover, 
the use of quantitative data may increase depending on the possibilities of quantifying the interacting factors in the iterative modeling cycle. The apparatus of signed graphs allows formally making forecasts of the development or trajectory of the simulated system in the phase space of its variables (factors) based on information about the structure and programs of its development by approximating them with pieces of trajectories of impulse processes on signed digraphs. Modeling using the graph theory makes it possible to remove uncertainty associated with predicting the development of a complex system and to propose the option of controlling a stochastic process.

The ultimate goal of structural modeling of the industrial cluster functioning is to develop structurally stable systems and scenarios for their sustainable development. An important feature of the model is the fact that the apparatus of signed graphs can give only a qualitative result. Therefore, time is defined not as a physical quantity, but as a sequence of occurrence of events called expertly significant.

The topic of modeling the resources of branch and regional processes is highly elaborated, but some aspects are insufficiently studied and require updating. So, scientists consider the problem of developing a methodology for modeling and studying the functioning of complex systems, taking into account the development of new information technologies.

\section{Modeling a cluster system}

\subsection{The algorithm for modeling a cluster system}

The algorithm for making a model of socio-economic development of the region's system can be represented in the form of a scheme (Table 1).

Table 1. The algorithm for making a model of the socio-economic system of the region

\begin{tabular}{|l|l|}
\hline Stage & Process content \\
\hline Stage I & $\begin{array}{l}\text { Analysis of the socio-economic system and the formation of numerous vertices of } \\
\text { the graph }\end{array}$ \\
\cline { 2 - 2 } & Dividing the vertices into groups: economic, social and environmental ones \\
\cline { 2 - 2 } & $\begin{array}{l}\text { Formation of variables that graphically display the relationship of the basic factors } \\
\text { of socio-economic development of the system }\end{array}$ \\
\hline Stage II & $\begin{array}{l}\text { Estimation of variables reflecting the relationship of the basic factors of socio- } \\
\text { economic development of the system }\end{array}$ \\
\cline { 2 - 2 } & $\begin{array}{l}\text { Estimation of the weights of the digraph arcs, relationships of basic factors and } \\
\text { forming a system of indicators }\end{array}$ \\
\cline { 2 - 2 } & Impulse exposure selection \\
\cline { 2 - 2 } & Composing an adjacency matrix and calculating the degrees of a vertex \\
\hline Stage III & Model-based system management \\
\hline Stage IV & $\begin{array}{l}\text { Work with the digraph model, the relationship between the basic factors of the } \\
\text { socio-economic development of the system }\end{array}$ \\
\hline Stage V & $\begin{array}{l}\text { Development of a strategic graphic image of the relationship of the basic factors of } \\
\text { the socio-economic development of the system }\end{array}$ \\
\hline
\end{tabular}

At the initial stage, information is collected about the studied socio-economic system. Then, certain concepts and assumptions regarding the system are formulated and, finally, a mathematical model is worked out that allows evaluating the dynamics of the system's behavior (development).

Forming a model of socio-economic functioning and development of the cluster is based on the estimation of the proposed structures and the interconnections of the basic factors according to their degree of compliance with the principle of proportionality of 
elements, which provides for the balance of various components of socio-economic systems.

The results of functioning of the system components are determined by a large number of variables interacting with each other, reacting with different sensitivity to changes in the surrounding variables. Therefore, in order to develop proposals for the improvement of the system, in mathematical modeling of its functioning, it becomes necessary to find a compromise between the accuracy of modeling results and the ability to obtain reliable information about the dynamics of changes in factors necessary to build an adequate model.

When creating a graph, first of all, a set of vertices, where control actions should be introduced leading to a desired result, as well as the nature of these actions are determined. These data are the basis for working out detailed management plans for the socio-economic system.

In the model of functioning of an industrial forest cluster, a set of vertices is divided into several groups (Table 2).

Table 2. The sets of vertices; indicators and management tools of the model

\begin{tabular}{|l|l|}
\hline Name & Indicators \\
\hline I Economic & $x_{1}-$ Volume and quality of production \\
& $x_{2}-$ Depreciation of funds \\
& $x_{3}-$ Cost of production \\
& $x_{4}-$ Volume of raw materials \\
& $x_{5}-$ Transport accessibility, construction of roads and industrial \\
& infrastructure \\
\hline II Financial & $x_{6}$ - Export and import balance \\
& $x_{7}-$ Investments \\
& $x_{8}-$ Credit policy (availability of loans) \\
& $x_{9}-$ Tax collection \\
& $x_{10}-$ Demographic policy (attracting migrants) \\
\hline III Social & $x_{11}-$ Highly qualified jobs \\
& $x_{12}-$ Cooperation with scientific and educational organizations \\
& (research) \\
& $x_{13}-$ Information support to the cluster \\
& $x_{14}-$ Development of social infrastructure \\
& $x_{15}-$ Population decline (migration) \\
\hline IV Environmental & $x_{16}-$ Volume and quality of the raw material base \\
& $x_{17}-$ Reforestation \\
& $x_{18}-$ Treatment facilities \\
& $x_{19}-$ Waste recycling \\
& $x_{20}-$ Pollution level \\
\hline Indicators & $x_{21}-$ Incomes of the population \\
& $x_{22}-$ Credit debt \\
\hline Management tools & $x_{23}-$ Share of high-tech production \\
& $x_{24}-$ Tax burden \\
& $x_{25}-$ Legal restrictions \\
& $x_{26}-$ Technological update \\
\hline
\end{tabular}

Determining the nature and points of introduction of control actions is the initial stage in the development of detailed management plans. To link these plans to specific conditions, it is necessary to know when to introduce them. The task is to determine the time to influence the socio-economic system to achieve certain goals. The choice of points and the nature of impact on the formation of a socio-economic model are extremely complex tasks, the solution of which is based on a complex of multiple modeling methods.

After forming numerous basic factors that correspond to the vertices of the signed digraph and describing their mutual influence, a graphic image of the relationship between 
the basic factors of socio-economic development is made. In addition, macroeconomic level factors are introduced and are included in the model after considering the combination of models.

Modeling the mutual influence of the basic factors of the system allows:

- displaying the mutual influence of the basic factors that have both direct and indirect effects on the parameters of the system;

- supplementing this model with factors of macroeconomic and social levels.

Formalization connects the parameters of the socio-economic system, which allows to manage socio-economic development $[6,7]$, and, therefore, influence the final results of the regional economy. Using the selected system of indicators in the modeling process will make it possible to determine the functional dependencies between the mentioned indicators and, as a result, to make an objective assessment of the cluster potential. The sequence of events is the scenario of the behavior of the socio-economic system, and the sequence of introducing control actions is the management scenario [8]. Using the developed system of indicators in the modeling process makes it possible to determine the functional dependencies between the selected indicators and, as a result, objectively assess the development potential of the cluster and the mutual influence of factors of its socioeconomic development.

Thus, from the traditional tasks for this modeling apparatus ("what will happen if"), a transition to solving a more substantial problem is possible: "what needs to be done to achieve the given system behavior".

\subsection{The process of modeling a cluster system}

The modeling process is gradual. At each step, data is collected from the monitoring vertices to identify the expertly significant events. Having identified the expertly significant events, the first management program is calculated. If its calculation is not possible, then the next situation is selected for calculation. After obtaining the management program, the introduction of control actions into the model of the socio-economic system takes place.

When forming control actions, it is reasonable to assume that the government is capable, within certain limits, of directly affecting the amount of investments, expenditures on social programs, the money supply, the level of tax rates and other instruments of influence. For each of the considered types of regions, a totality of the necessary control actions is identified that ensures their effective development.

When modeling, the principle of stepwise increasing the complexity of the model is applied. Working out of the model and conducting the model experiment was performed with the programs using information stored in a database and updated interactively during communication with experts. Using the program it was possible to investigate the problems of observability, controllability, stability, complexity, connectivity, adaptability of systems, impulse processes, and catastrophes.

Currently, difficulties arise not only from the lack of information, but also from its redundancy. The main tasks that should be solved to provide information support to the research in the field of sustainable development are the following: to systematize information, conduct quantitative and qualitative analysis of data, informatively fill out socio-ecological-economic models in a form convenient for the researchers.

Using the proposed system of indicators in the modeling process will make it possible to determine the functional dependencies between the selected indicators and, as a result, to provide an objective assessment of the region's potential and its management capabilities. 


\subsection{Forecasting the cluster system model}

When creating a model, a compromise is necessary between the generality of the model and the possibility of a realistic assessment of its parameters (weight, delay, etc.) and their interpretation: it can be difficult to even explain the meaning of the weights $w(u, v)$ if $u$ or $\mathrm{v}$ is difficult to measure or precisely determine. That is why it is important to investigate the conclusions obtained only by considering the signs.

The advantage of the model in the form of a weighted digraph is that it allows to accurately formulate problems, for example, forecasting the need of the system in resources, or forecasting the impact of new technologies on this need. The problems of identifying and selecting possible alternative strategies can also be analyzed to meet the limitations associated with the requirements of cluster members, etc. To analyze the above questions, let us define that each vertex $u_{i}$ takes the value (or reaches the level of) $v_{i}(\mathrm{t})$ at discrete time instants $t=0,1,2, \ldots$ The rule for changing the values of the impulse process establishes how deviations of the values of the variables propagate in the system for some time. Thus, the forecasting problem can be formulated as follows: to predict the value $v_{i}(\mathrm{t})$ of the vertex $u_{i}$ at time $\mathrm{t}$ or, in other words, to predict a change in $v_{i}(\mathrm{t})$ for a given initial value of $v_{i}$ (ref).

There are several options for setting the forecast task:

— taking into account the impact of new technologies and other capabilities, by changing the weighted digraph at some point in time $\mathrm{T}$, when this technology will be introduced;

- probabilistic forecasts of the expected value $M\left(v_{i}(t)\right)$ based on an estimate of the expected value of time $\mathrm{T}$.

- possibility to introduce restrictions or standards related to requirements to the quality of the environment, as well as restrictions of a different origin, giving a vertex a boundary within which the values of its corresponding parameter change (this is done by introducing two vectors $=\left(m_{1}, m_{2}, \ldots, m_{n}\right)$ and $M=\left(M_{1}, M_{2}, \ldots, M_{n}\right)$ representing the lower $m_{i}$ and upper $M_{i}$ constraints for each vertex $\left.\mathrm{M}\right)$.

When the constraints are set, it is possible to find options for strategies that satisfy them. The strategy is a procedure that leads to a change in the system. If the system is presented as a weighted digraph, some possible changes or strategies are as follows:

- to change the values of a number of vertices at a given time;

- to add a new vertex (factor) and new arcs to and from it (which correspond to the relations of interaction of this factor with those already in the model) at a certain time;

- to change the sign of any of the arcs at a certain time;

- to change the weight of any of the arcs at a certain time;

- to add a new arc to the digraph between the existing vertices;

— to add a new contour (reinforcing or reducing the deviation).

Another option is to find a policy that maximizes (or minimizes) the values of some vertices (for example, vertices that measure the quality of "poorly trained students") if there are restrictions on the values of some other vertices. It is important to find not the strategies that satisfy the constraints, but the strategies that do not allow any variable to take too large or small values, i.e. stabilizing strategies.

The concept of sustainability can be clarified using the model of propagation of changes in the values of variables. Let us introduce assumptions about the effect of changing the parameter value in one vertex on the parameters of the remaining vertices of the graph the value change rule for the parameters of the vertices. Assuming that the basic data (for example, the initial values of the parameters at each vertex and the weight of the arcs) are not known exactly, the final conclusions based on a certain rule for changing the values of the parameters of the vertices will also be probabilistic. Therefore, any result obtained with the model should be taken as preliminary; a "sensitivity analysis" should be carried out for 
it, which includes repeated modeling with the changed basic data, as well as the use of modified rules for changing the values of the vertex parameters.

In a signed digraph the vertices are represented by the vector $u_{1}, u_{2}, \ldots, u_{n}$. and take the values $v_{i}(\mathrm{t})$ at discrete time instants $\mathrm{t}=0,1,2, \ldots$, and changes (1) for $\mathrm{t}>0$ are called impulses.

$$
p_{j}(\mathrm{t})=v_{i}(\mathrm{t})-v_{i}(\mathrm{t}-1)
$$

If we set the initial condition at $\mathrm{t}=0$ and introduce the notation:

$$
\operatorname{sgn}\left(u_{j} u_{i}\right)=\left\{\begin{array}{c}
1, \text { if the } \operatorname{arc}\left(u_{j} u_{i}\right) \text { is positive } \\
-1, \text { if the } \operatorname{arc}\left(u_{j} u_{i}\right) \text { is negative } \\
0, \text { if the } \operatorname{arc}\left(u_{j} u_{i}\right) \text { is absent }
\end{array}\right.
$$

then for $\mathrm{t} \geq 0$, the value change rule will be as follows:

$$
v_{i}(\mathrm{t}+1)=v_{i}(\mathrm{t})+\sum_{j=1}^{n} \operatorname{sgn}\left(u_{j} u_{i}\right) p_{j}(\mathrm{t})
$$

The condition (3) for an autonomous impulse process in a signed digraph is generalized to the rule that determines the changes in the values of the vertices of the weighted digraph for an autonomous impulse process (in fact, a system of finite difference equations with parameters $\left.w\left(u_{j} u_{i}\right)\right)$. Let us assume that

$$
v_{i}(\mathrm{t}+1)=v_{i}(\mathrm{t})+\sum_{j=1}^{n} w\left(u_{j} u_{i}\right) p_{j}(\mathrm{t})
$$

where $\mathrm{w}(\mathrm{u}, \mathrm{v})=0$ if the $\operatorname{arc}(\mathrm{u}, \mathrm{v})$ is absent; if there is an arc from $u_{j}$ to $u_{i}$ with the weight $\mathrm{w}=w\left(u_{j} u_{i}\right) \neq 0$ and the value of the vertex $u_{j}$ increases at time $\mathrm{t}$ by $\mathrm{k}$ units, then as a result, the value of the vertex $u_{j}$ at time $(\mathrm{t}+1)$ increases by $k \times w$ units.

Using the definition of the impulse (1), the equation (4) can be written as:

$$
p_{i}(\mathrm{t}+1)=\sum_{j=1}^{n} w\left(u_{j} u_{i}\right) p_{j}(\mathrm{t})
$$

The rule for changing the values given by the equation (5) neglects time delays and assumes that each action occurs in a single time cycle.

We can associate $p_{j}(\mathrm{t})$ and $v_{j}(\mathrm{t})$ with elements of the adjacency matrix $\mathrm{A}$; and $\mathrm{G}$ is a weighted digraph. Then for a simple impulse process with the initial vertex $u_{i}$ :

$$
\begin{gathered}
p_{j}(\mathrm{t})=\left\{\text { element } i, j \text { of the matrix } A^{t}\right\} \\
v_{j}(\mathrm{t})=v_{j}(\mathrm{ref})+\left\{\text { element } i, j \text { of the matrix } I+A+A^{2}+\cdots+A^{t}\right\}
\end{gathered}
$$

In the vector notation, this rule, formulated for a simple impulse process with an initial vertex $u_{i}$ and $\mathrm{P}(0)=(0,0, \ldots, 0,1,0, \ldots, 0)$, has the form of $P(t)=P(o) A^{t}$

The equation means that $\mathrm{P}(\mathrm{t}+1)=\mathrm{P}(\mathrm{t}) \mathrm{A}$

In this way, the forecast problem is solved, and the above statements give a solution for the first statement of the problem of choosing strategies. If the desired value (the set of values) of the vertex $u_{J}$ (the set of vertices) is given, then the sums of the powers of the matrix A are simply calculated until at least one element $i, j$ takes the desired value. The initial impulse directed to the vertex $u_{i}$ leads to the solution. Similarly, a solution should be calculated if more complex strategies are used, or least cost strategies are desired.

Checking the stability of a digraph reduces to examining simple questions about its eigenvalues. If a weighted digraph $\mathrm{G}$ is impulse-stable for all simple impulse processes, then each eigenvalue of the vertex adjacency matrix, which sets it in an absolute value, does not exceed 1. The opposite formulation is usually used: if a weighted digraph $G$ has its eigenvalue exceeding 1 in modulus, then $G$ is impulse-unstable for any simple impulse process. If an integrally weighted digraph is impulse-stable for any simple impulse processes, then each of its non-zero eigenvalues is equal to 1 in the absolute value. Therefore, weighted digraph is impulse-stable for all simple impulse processes. If a nonzero eigenvalue is multiple, then the statement is not applicable. A weighted digraph $G$ is absolutely stable for any simple impulse process in the case when $\mathrm{G}$ is impulse-stable for any simple impulse process and there is no eigenvalue equal to 1 among G's eigenvalues. 


\section{Expert selection of factors for the formation and development of forest clusters}

One of the important problems in creating a structural model of a cluster is the selection of factors for the formation and development of industrial clusters in the regional economy and practical recommendations for optimizing the organizational mechanisms for managing this socio-ecological-economic system. To identify these factors, assumptions and prospects that influence the formation of a forestry cluster in Arkhangelsk Oblast, statistical (regression-correlation) analysis methods, questionnaires and interviews with experts were used. Expert methods are widely used in the study of socio-economic and political phenomena and processes in those cases when there is lack of reliable statistical information about the phenomenon being studied or ideas about the conditions of its functioning are vague. Also, expert methods are used with a shortage of time to conduct research on this phenomenon or if it is performed in extreme situations.

Expert research methods [9] usually take the form of surveys (in our case, oral interviews) or written questionnaires. For example, to make a quantitative assessment of poorly formalized factors, an oral interview with experts was used. There was direct contact with respondents, their attitudes to the discussed problem were recorded, and the conversation was monitored and directed. In this case, psychological pressure on experts is not allowed, they freely express their opinions. Highly qualified specialists working for the government of Arkhangelsk Oblast were brought in as experts. Based on their knowledge and many years of experience, they expressed their opinions, assessments, and proposals regarding the features of functioning of industrial clusters. Their opinions are related to each other (a statistically significant coefficient of concordance is $W=0.76$ ). The interview consisted of several iterations, each of them began with an individual interview with experts. At the first step, the experts freely expressed their opinions without any argumentation; at the second step, they were asked to substantiate their conclusions. Each of them was informed of the generalized data obtained by processing the materials obtained at the first step. In this way, an absentee and anonymous expert discussion was organized. At the third step, the experts could correct some of their judgments under the influence of the new materials presented, justifying their changed judgments and conclusions. The first step made it possible to indentify the factors to be included in the model, while the last step helped to rank them.

\section{Programming a forest cluster digraph}

Johnson's algorithm was used to create a program that finds all possible digraph cycles. It should be noted that this algorithm is not the only one that allows analyzing the structure of digraphs. Several algorithms are described in scientific works that find elementary circuits of any direct graph: Tiernan's, Weinblatt's, Tarjan's (the variation of Tiernan's algorithm), Ehrenfeh's, Fosdick's and Osterwale's algorithms. The screen displays the total number of the cycles found, a numbered list of the cycles and indicators of the "balance" of the graph. The graph is balanced if $b=1(0<b \leq 1), a=\infty(0<a<\infty)$. The presence of a significant number of contours in the digraph, reinforcing the deviation and many contours that counteract the deviation, leads to its instability due to the rapid growth of values in individual vertices and due to an increase in fluctuations.

To make a conclusion about the impulse stability of a digraph, we used the theorem concerning the relation between the impulse stability of digraph $G$ and the eigenvalues of the adjacency matrix of the vertices of this digraph. At the next step, the program displays complex eigenvalues of the adjacency matrix of digraph vertices in an algebraic form and 
the values of their modules. The eigenvalue modules exceed 1 , which indicates the impulse instability of the graph.

To verify this, it is necessary to direct the impulse to the vertex, enter the coordinates of the vector, the number of iterations, and the program displays the value of the impulse $p(t)$ at each moment of time and the vector of digraph vertices (the initial values of this vector are assumed to be zero: $v(t=0)=(0 ; 0 ; 0 ; 0 ; 0 ; 0 ; 0))$. Thus, the forecast problem is solved on the basis of the constructed model (Fig. 1).

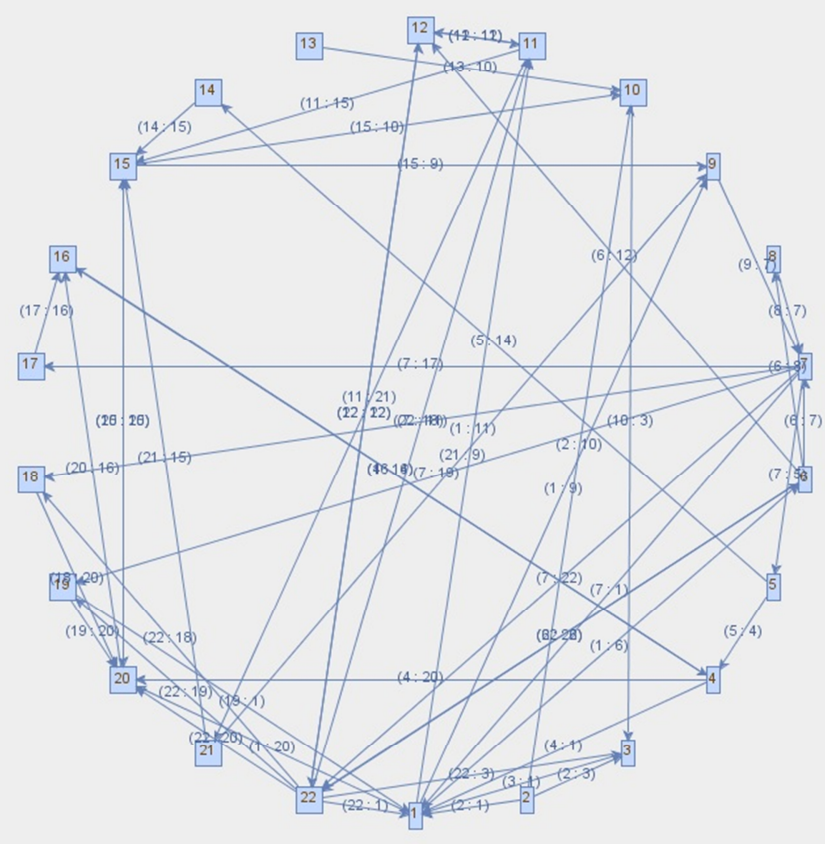

Fig. 1 Graph visualization

The model (Fig. 1) contains the variables:

$x_{1}-$ Volume and quality of production

$x_{2}-$ Depreciation of funds

$x_{3}-$ Cost of production

$x_{4}-$ Volume of raw materials

$x_{5}-$ Transport accessibility, construction of roads and industrial infrastructure

$x_{6}-$ Export and import balance

$x_{7}-$ Investments

$x_{8}-$ Credit policy (availability of loans)

$x_{9}-$ Tax collection

$x_{10}-$ Demographic policy (attracting migrants)

$x_{11}-$ Highly qualified jobs

$x_{12}$ - Cooperation with scientific and educational organizations (research)

$x_{13}$ - Information support to the cluster

$x_{14}$ - Development of social infrastructure

$x_{15}$ - Population decline (migration)

$x_{16}-$ Volume and quality of the raw material base

$x_{17}-$ Reforestation

$x_{18}$ - Treatment facilities

$x_{19}-$ Waste recycling 
$x_{20}-$ Pollution level

$x_{21}$ - Incomes of the population

$x_{22}$ - Share of high-tech production.

\section{Final remarks}

Currently, difficulties arise not only from the lack of information, but also from its redundancy. The main tasks that should be solved to provide information support to the research in the field of sustainable development are the following: to systematize information, conduct quantitative and qualitative analysis of data, informatively fill out socio-ecological-economic models in a form convenient for the researchers.

Modeling using the graph theory makes it possible to remove uncertainty associated with predicting the development of a complex system and to propose the option of controlling a stochastic process. The ultimate goal of structural modeling of the forest cluster functioning is the development of structurally stable systems and scenarios of their sustainable development. An important feature of the model is the fact that the apparatus of signed graphs can give only a qualitative result, impulse processes, and disasters.

Using the proposed system of indicators in the modeling process will make it possible to determine the functional dependencies between the selected indicators and, as a result, an objective assessment of the region's potential and its management capabilities.

The research was carried out with the financial support from the Russian Foundation for Basic Research within the framework of scientific project No. 18-010-00147 "Theoretical and methodological approaches to the functioning of the territorial production timber industry cluster as a tool for the spatial development of the Russian Federation".

\section{References}

1. R.J. Chilundo, G.A. Maúre, U.S. Mahanjane, Journal of Cleaner Production 23820 (2019). Available at: https://doi.org/10.1016/j.jclepro.2019.117878

2. Amir Fateh, Farzad Hejazi, Mohd Saleh Jaafar, Izian Abd. Karim, Azlan Bin Adnan, Soil Dynamics and Earthquake Engineering 80 (2016). Available at: http://dx.doi.org/10.1016/j.soildyn.2015.10.009

3. L. C. S. Martinho, J. V. C. Vargas, W. Balmant, J. C. Ordonez, International Journal of Refrigeration 68 (2016). Available at: http://dx.doi.org/10.1016/j.ijrefrig.2016.04.023

4. Qinhua Wang, Weidong Fu, Shuzhi Yu, Luke Allan, Ming Gu, Journal of Wind Engineering and Industrial Aerodynamics 179 (2018). Available at: https://doi.org/10.1016/j.jweia.2018.06.007.

5. Truls Flatberg, Vibeke S. Nørstebø, Knut Bjørkelo, Rasmus Astrup, Nils Egil Søvde, Forest. Policy and Economics 92 (2018). Available at: https://doi.org/10.1016/j.forpol.2018.04.008.

6. J. Buongiorno, International Journal of Forecasting 12 (3) (1996)

7. M. Pecchi, M. Marchi, V. Burton, F. Giannetti, G. Chiric, A literature review. Ecological Modelling 4111 (2019). Available at: http://dx.doi.org/10.1016/j.agrformet.2006.01.012

8. M. Ya. Antonovsky, R. A. Fleming, Yu. A. Kuznetsov, W. C. Clark, Theoretical Population Biology 37 (2) (1990).

9. T. Agasiev, A. Karpenko, Procedia Computer Science 1032017 (2017). DOI: 10.1016/j.procs.2017.01.120 